\title{
A. Lisíchkina
}

\section{Estrategias de formación de la comprensión lectora en la enseñanza de la lengua (Primaria, Secundaria, Superior)}

\author{
Alexandra Lisíchkina, \\ profesora del Departamento del Español \\ de la Universidad MGIMO, Moscú, Rusia. \\ sasha.lisichkina@rambler.ru
}

\begin{abstract}
Resumen. En el artículo se trata de la formación de la comprensión lectora, así como de la importancia de los textos en el proceso de la enseñanza de lenguas extranjeras. En el artículo aparecen ejemplos de textos y tareas que demuestran diferentes estrategias que usan los españoles para impartir clases de idioma. Los ejemplos son de varios libros de español como lengua extranjera y como lengua materna. Todos los libros han sido ediłados en España. Los ejemplos de las tareas textuales se analizan para comprender si hay coincidencias y diferencias entre las estrategias para enseñar el español usadas en Rusia y España, lo que hace posible la posterior aplicación de éstas en clases de español que se imparten en la universidad MGIMO.
\end{abstract}

Palabras clave: lectura, enseñanza de ELE, comprensión lectora, estrategias y tareas, texto.

\section{Alexandra Lisíchkina}

\section{Strategies for the formation of reading comprehension in teaching of the language (Primary, Secondary, Superior)}

\begin{abstract}
The article is concerned with the importance of the using of texts in the process of studying of foreign languages. The article gives an analysis of exercises for the texts. Attention is given to the differences between the exercises we use in the Russian and in the Spanish education systems.

Key words: reading, teaching of Spanish, reading comprehension, strategies and exercises, text.
\end{abstract}

El objetivo de la investigación es valorar la importancia de la lectura en el proceso de la enseñanza del español como lengua materna y como segunda lengua y analizar los tipos de ejercicios que aparecen en los libros de español editados en España.

\section{Comprensión lectora en la enseñanza de la lengua}

Al comenzar, habría que decir que la lectura es el principal medio de aprendizaje de todo tipo de asignaturas. La lectura es el mecanismo que nos da acceso a la mayor parte de la información existente en el mundo, puesto que el conocimiento se acumula en libros y enciclopedias.

En lo que se refiere a la metodología de enseñanza de la lengua, la lectura presenta interés como medio del desarrollo de capacidades cognitivas superiores, tales como la reflexión, la conciencia. Si en Primaria las clases de lectura consisten en aprender a descifrar y descodificar lo escrito, en Secundaria se pretende comprender, interpretar, establecer propias opiniones formuladas como valoraciones y juicios, comentar 
y analizar el texto escrito. Esta habilidad debe desarrollarse hasta que los alumnos sean capaces de comprender y analizar otras materias que se imparten durante el proceso de la enseñanza. Incluso, en algunos casos, en idiomas extranjeros. Y en el caso de la universidad MGIMO, en clases del "lenguaje profesional", cuyo objetivo es la preparación lingüística de un profesional que sea capaz de ejercer sus funciones en la lengua extranjera sin acudir al intérprete [4, c. 108-118].

Por eso tiene valor especial la comprensión lectora, cuyo desarrollo supone el desarrollo de la competencia de la comunicación lingüística.

Cabe mencionar que a pesar de que escribir y hablar es lo que se produce durante la comunicación y leer es lo que se percibe, las dos primeras actividades se aprenden mediante el proceso de leer. Eso se debe a que el lenguaje escrito suele ser más rico y normativo que el del habla corriente. De ello proviene la convicción de que para saber escribir y hablar correctamente es indispensable leer lo más posible, y no sólo las obras literarias, sino también la prensa e, incluso, foros y charlas en Internet, ya que de éstos se puede aprender las nuevas tendencias que aparecen en la lengua, tanto en el léxico como en la gramática.

En la comunicación habrá ejemplos de tipos de tareas destinadas a desarrollar la comprensión literaria en los estudiantes de español.

Se sabe que hay varios enfoques de enseñar una lengua extranjera: directo, comunicativo, intensivo. Cabe mencionar que para enseñar una lengua extranjera se utilizan los métodos ya conocidos y a la vez se desarrollan métodos nuevos e intensivos [5, c. 17-22]. Hay que añadir que en el mundo actual se opta por los que estimulen la actividad mental. Esta estrategia se realiza perfectamente con la utilización de los textos. Este concepto lo podemos encontrar en los libros españoles para estudiantes del español como lengua extranjera. Allí siempre vemos tales tipos de ejercicios después de los textos, como: "puedes explicar por qué en unas frases se usa el modo indicativo y en otras, el subjuntivo" o "has deducido por qué en estas frases aparece el futuro perfecto?" Está claro que nosotros también tratamos de utilizar tales estrategias con nuestros estudiantes, porque así contribuimos a que recuerden lo estudiado y aprendan a hacer deducciones. Es obvio que este tipo de tarea no se aplica a cualquier tema, pero de todas formas se puede usarlo en varios casos durante el aprendizaje. Por ejemplo, incluso en el nivel inicial es posible trabajar a base del texto con el mínimo del material estudiado. En la etapa inicial de aprendizaje después de leer y traducir el texto, para verificar la comprensión, el profesor puede formular preguntas a base del contenido del texto que incluyan todos los aspectos formales trabajados [8, p. 229-244].

Para analizar diferentes tareas y estrategias que se utilizan en los libros españoles hemos decidido acudir a libros escritos para distintos niveles de conocimiento de la lengua, algunos libros de español como lengua extranjera y otros - para los nativos, o sea, español como lengua materna. Los libros que hemos usado son tres: uno de nivel B2 para adultos que estudian español como lengua extranjera [1], otro de cuarto año de primaria que es un libro de español para españoles [2], y el último es con el que estudian en primer curso de Bachillerato, que también es un libro de español como lengua materna [3]. Hemos tratado de estudiar varios tipos de textos, tales como textos narrativos y periodísticos, ya que el lenguaje varía según el tipo de texto, por lo que las tareas también pueden ser diferentes.

Como nosotros damos clases de español como lengua extranjera, hemos decidido empezar con el libro de español ELE del nivel B2 para adultos, y ver textos y tareas que están dirigidas a la formación de la comprensión lectora. Es un libro editado en España y se supone que lo usarán extranjeros que tengan este nivel. Como es sabido que semejantes libros pretenden enseñar la lengua mediante el método comunicativo, en ellos a menudo aparecen textos introduciendo cualquier tema, habitualmente gramatical. Como ejemplo hemos elegido un texto cuyo tema es la ecología. La elección se debe a que es uno de los temas básicos para nuestra universidad, que enseñamos usando los libros de niveles desde A1 hasta B2 [6; 7].

Pongamos como ejemplo un texto periodístico, en él vemos unas palabras subrayadas, que son conectores. La primera tarea que sigue el texto es "poner en cada columna las palabras y expresiones en negrita que le dan cohesión al texto". Cabe mencionar que tales tareas no suponen traducción, ya que suelen ser usadas en grupos de estudiantes de diferentes países o con un profesor nativo, por eso los estudiantes se ven obligados a deducir el significado de cada uno de los conectores, según el contexto. En la siguiente tarea le ofrecen al estudiante unos cuantos conectores más, la mayoría de los que ya los sabe, y hay que introducirlos en las mismas columnas. Lógicamente luego se le pide al estudiante cambiar los conectores del texto por otros equivalentes. Tal tarea nos parece útil, ya que los sinónimos enriquecen el lenguaje y hacen el habla más completa y variada. Otra tarea también se debe a la clase típica de unos cursillos donde se usan tales libros; hay que escribir dos frases para que el compañero las una, utilizando algún conector. En general cualquier tarea que exige que el estudiante invente algo, es indispensable en el proceso de aprendizaje de un idioma, puesto que se necesita aplicar directamente lo que se está estudiando, y esto, claro, es lo que pretendemos nosotros - que los estudiantes utilicen el material que les impartimos. En 
la misma unidad luego aparece un ejercicio que consiste en redactar un informe sobre un tema ecológico "seleccionando los conectores que vas a utilizar". Al analizar estas tareas vemos que aquí en el texto se introduce un tema gramatical; el texto es el ejercicio principal, puesto que se aprende a usar los conectores poniéndolos en el texto; y además es un ejemplo para redactar un texto parecido utilizando marcadores del discurso. Aquí se puede añadir que en los libros escritos por los españoles se suele prestar especial atención a los conectores en todo nivel de conocimiento del idioma.

No podemos decir exactamente, si hay que estudiar los sinónimos sin traducción, pues sabemos que en la lengua no hay sinónimos completos. Pero de todos modos saber explicar en el idioma extranjero el significado de un concepto es saber usar el idioma no sólo para realizar tareas convencionales. Por otro lado, este tema léxico nos recuerda la importancia de los conectores y marcadores del discurso en un lenguaje rico.

El siguiente libro que hemos elegido para analizar los textos y tareas es un libro para el cuarto año de Primaria, o sea, para los nativos. Por supuesto, en este libro todos los textos contienen temas actuales para esta edad, es decir, hay más textos sobre, por ejemplo, animales que sobre la ecología, como en el ejemplo anterior. A pesar de esto, nos parece útil ver cómo se usan los textos en todas las etapas de la enseñanza de la lengua. Además, el tema léxico de la unidad que hemos elegido es flora y fauna - tema que también estudiamos en nuestras clases.

Cada lección empieza con un texto que es la base de todas las tareas que hay en la lección. Los primeros ejercicios sirven para controlar la comprensión del texto: son preguntas sobre el contenido. La parte léxica de la lección se introduce con un dibujo que se refiere al texto, pero el tema, que comprende las terminaciones del gentilicio, no tiene nada que ver con el propio texto. La parte de ortografía en esta lección trata el acento gráfico. La relación con el texto está en el contenido de éste: el texto narra sobre los animales y plantas, pues todas las palabras que hay para practicar las reglas de acentuación son nombres de animales o plantas: hay nombres de representantes de flora y fauna que están en diferentes columnas según la sílaba acentuada, es decir, agudas, llanas y esdrújulas. A propósito, a estas columnas hay que añadir más animales y plantas. Y eso es lo que atrae nuestra atención - lo frecuente que los libros españoles piden que el estudiante piense, recuerde, invente.

Toda la lección y todos los tipos de ejercicios que hay se basan en el texto que rige el contenido de la lección. Como hemos notado, en los libros editados en España el texto no suele ser ejemplo de cómo se utiliza una u otra regla, sea ésta gramatical u ortográfica, por ejemplo. Sino que el texto es lo que introduce cualquier regla o información nueva y de allí aparecen todas las conclusiones, independientemente de si están en el libro como una regla o si el mismo alumno debe deducirlas.

Pero claro, lo que nos interesa es analizar y comparar aquel material que ofrecen los libros españoles que pueda ser utilizado de alguna manera en nuestras clases. Por eso hemos prestado especial atención a los libros de la lengua castellana y literatura que se usan en Bachillerato, ya que el nivel de dificultad y los temas deben de tener algo que ver con el material impartido aquí a nuestros estudiantes en los últimos años de sus estudios. Hemos elegido un libro para el primer curso de bachillerato. Lo primero que hay que destacar es que el contenido de cada lección ya no es igual al que vimos en el libro para el cuarto año de primaria donde el texto introduce las reglas, aquí el texto no es base de toda la lección, sino la estructura que es más habitual para nosotros. En este caso la estructura consiste en que primero vienen temas y reglas gramaticales, léxicos y ortográficos y sólo después aparece el texto, que se presenta como ejemplo de todo lo dicho anteriormente y es fuente de tareas dirigidas a practicar lo estudiado. Por ejemplo, tales tareas como encontrar equivalentes, contestar a las preguntas, completar las frases del texto con artículos y preposiciones [6; 7].

Otro tema de interés es el tema de medicina, ya que en nuestros manuales también está presente este tema. Desde nuestro punto de vista lo primero que se nota es que la primera tarea después de cada texto es definir el asunto o tema general del texto o señalar cuál es la idea global. Nos parece curioso, pues esto muestra que la estrategia principal es que los estudiantes primero analicen todo el texto y sólo después pasen a tareas más concretas. Si lo pensamos se comprende la causa de que en nuestros manuales las preguntas sobre el texto son más concretas. Este esquema es indispensable para que el estudiante repita frase por frase el texto, pase el estilo directo al estilo indirecto, cambie las formas verbales, use el léxico. La idea es que así ya se memorizan las estructuras gramaticales y palabras nuevas. Además hay que añadir que la tarea de contestar a las preguntas después de leer un texto, supone un cambio de actividades, lo que hace que el estudiante realice varias tareas durante una clase y con el mismo material léxico y gramatical. Por otro lado, las preguntas más generales se concentran en que se haga un resumen del texto.

Otro aspecto interesante es que se pide explicar el significado de algunas expresiones que aparecen en el texto. Se trata de frases donde se puede encontrar un tipo de juego de palabras, como por ejemplo "Los 
coches del siglo XIX se movían por la potencia de los caballos, y los nuestros se mueven por los caballos de potencia”. Se ve que una tarea parecida invoca a que se desarrollen las capacidades analíticas del lenguaje y de juego de palabras en los estudiantes. Y claro, aquí no se debe olvidar del nivel que, como ya lo hemos dicho, es relativamente alto y ya permite a los estudiantes realizar tales tareas.

Además hay tareas que piden clasificar algunas palabras del texto según la forma y la categoría gramatical, analizar el proceso de formación de palabras de la misma raíz o formar la familia léxica de palabras. En nuestra opinión este tipo de tarea es necesario para aprender un idioma extranjero también, ya que contribuye a que el estudiante deduzca y analice lo que estudia. Se puede suponer que cada uno de nosotros cada día enfrenta la situación cuando el estudiante no sabe deducir el significado de una palabra nueva, a pesar de que le es conocida la raíz de la palabra, por lo que podemos concluir que semejante tarea puede y debe ser aplicada en el aprendizaje de una lengua extranjera no sólo en los últimos cursos sino también en los primeros cuando los estudiantes adquieren sus primeros conocimientos sobre el idioma extranjero.

Conviene señalar que no es fácil encontrar un texto literario en libros de español para Bachillerato, suponemos que esto se debe a que el nivel de conocimiento de la lengua supone análisis de textos periodísticos porque estos muestran los cambios actuales en el idioma, que pueden ser tanto léxicos, como gramaticales. Otra razón puede ser la que los temas que se discuten en los periódicos son los más actuales y palpitantes. Es cierto que el lenguaje de los artículos de opinión que se publican en los periódicos refleja todo lo nuevo que pasa con un idioma. Además siempre está presente el aspecto estilístico; cualquier artículo de opinión, sobre todo si ha sido escrito por un escritor profesional, está lleno de todo tipo de recursos estilísticos: metáforas, comparaciones, alusiones, etcétera. Podemos hacer referencia al texto y tareas analizados arriba, pues es un buen ejemplo del uso de un texto periodístico en el que se emplean los recursos estilísticos ofrecidos para sacar el máximo aprovechamiento a la hora de aprender un idioma.

De todos modos, como ya lo hemos mencionado, la situación con los textos literarios no es tan favorable, y el único fragmento que proponemos al análisis es un pequeño fragmento de una novela de Eduardo Mendoza que en este libro aparece en la parte gramatical del libro, y el fragmento se propone para practicar el tema de estilo directo e indirecto, aunque la forma tampoco es habitual para nosotros. El caso es que no tenemos que pasar un diálogo al estilo indirecto, lo que supone ser la tarea esencial para este tema gramatical, la tarea consiste en señalar dos formas diferentes de reproducir el diálogo y después escribir de nuevo el texto empleando únicamente el estilo directo y con rayas, o sea el clásico estilo directo. Por supuesto, una tareal igual de analítica es posible sólo cuando el estudiante dispone de un nivel bastante alto de la lengua. En cualquier caso, nos parece útil encontrar diferencias y similitudes en las estrategias usadas en el sistema de aprender español como lengua materna y en el sistema que usamos nosotros, ya que así se puede aprender algo nuevo y tal vez aplicarlo en el futuro.

Al analizar la trascendencia de la lectura en clases de lengua y al observar los posibles ejercicios y estrategias basados en lo leído que se aplican para estimular en los estudiantes la competencia lingüística, se puede sacar la conclusión de que la comprensión lectora, siendo el principal medio de aprendizaje, se distingue por sus objetivos, estrategias y formas de trabajo en diferentes etapas educativas. Y esto depende no sólo de la edad de los estudiantes, sino de su nivel de dominio del idioma, asimismo del hecho de que la lengua estudiada es la lengua natal o extranjera.

En cualquier caso, la idea del presente artículo es que a pesar de que estamos convencidos de que el sistema ruso de enseñar lenguas extranjeras trae altos resultados, nunca se niega a que podamos mejorar y perfeccionar algo, por eso creemos que es útil analizar cómo se imparten los idiomas, en nuestro caso el español, en otros países y sobre todo en España, ya que los españoles sabrán cómo enseñar su lengua. Decimos que es útil porque estamos seguros de que siempre se puede aprender algo nuevo para aplicarlo después en nuestra actividad profesional.

\section{Literatura}

1. Borobio V., Palencia R. Nuevo ELE avanzado. SMEE, 2003. - Madrid. - P. 74. 175 p.

2. M. C. González Casado, Ch. González Parra, B. Oro. Trotamundos 4 Primaria. Equipo SM, 2009. - Madrid. P. 22-23. 223 p.

3. Hernández G., Cabrales J. M.. Lengua castellana y literatura // Bachillerato 1. SGEL, 2015. - Madrid. - Pp. 48, 137. $320 \mathrm{p}$.

4. Горенко Г. М. Актуальные вопросы преподавания языка специальности // Филологические науки в МГИМО. - М.: - Изд-во МГИМО-Университет, 2007. - С. 108-118. 
5. Гринина Е. А. Лингводидактика в неязыковом вузе: “Español actual A1/A2” // Вопросы иберо-романистики. - М.: МАКС Пресс, 2013. - С. 17-22.

6. Гринина Е. А., Микаэлян Ю. И. Español actual A1/A2. Современный испанский язык. Начальный курс. М.: Филоматис, 2013. - 192 с.

7. Гринина Е. А., Микаэлян Ю. И. Español actual В1. Современный испанский язык. Продолжающий курс. М.: Филоматис, 2015. - 204 с.

8. Gúseva I. Peculiaridades de la enseñanza de E/LE en Rusia: metodología y materiales didácticos // Actas del XXXVIII Congreso Internacional de la Asociación Europea de Profesores de Español; AEPE, 2003. - Pp. $229-244$. 